\title{
Antagonistic pleiotropy and the evolution of wing dimorphism in the sand cricket, Gryllus firmus
}

\author{
D. A. Roff
}

At $30^{\circ} \mathrm{C}$ the micropterous females of the sand cricket, Gryllus firmus, begin reproduction at an earlier age after eclosion and have a larger cumulative fecundity than macropterous females. These reproductive costs may offset the advantages of being macropterous and hence capable of migration. The evolutionary significance of this phenotypic trade-off, which is characteristic of wing dimorphic insects in general, is contigent on the traits being genetically correlated. The genetic basis of the phenotypic tradeoff between flight capability and reproduction in the sand cricket, Gryllus firmus, was examined by selecting for increased and decreased incidence of macroptery, and measuring the age schedules of fecundity of macropterous and micropterous females in the selected and control lines. The two traits, wing dimorphism and age schedule of reproduction, are shown to be genetically correlated. Although the mean fecundity within the selected populations changed the fecundities of macropterous and micropterous forms remained constant, suggesting that the age schedule of reproduction may itself be a threshold trait with respect to the continuously varying character controlling the expression of wing form. The relevance of antagonistic pleiotropy to the maintenance of genetic variation for wing form and the age schedule of reproduction is discussed.

\section{INTRODUCTION}

Migration by flight is an important aspect of the life history of many insect species, permitting them to colonize highly ephemeral habitats (Southwood, 1962; Johnson, 1969; Harrison, 1980; Dingle, 1985). However, the benefits of migration may be offset in part both by the energetic cost of flight and by the cost of producing the machinery of flight, viz the wing muscles, wings etc (Roff, 1977, 1986a; Roff and Fairbairn, 1990; Inglesfield and Begon, 1983; Denno et al., 1989). The latter cost has been demonstrated by experiments with wing dimorphic insects, (i.e., species in which some individuals within a population or family have functional wings and are capable of flight, while others have reduced wings and are incapable of flight). Within such species there is a consistent pattern of delayed reproduction and reduced fecundity of the fully winged morph (Roff, 1986a; Denno et al., 1989; Roff and Fairbairn, 1990). If genetically based, these trade-offs could significantly influence the evolution of the incidence of wing dimorphism within a population (Roff,
$1975,1990 a$ ). But though there is abundant evidence of a phenotypic trade-off between reproduction and wing morph, there are no studies demonstrating that these tradeoffs have a genetic basis and are thus examples of antagonistic pleiotropy.

The purpose of the present study was to test the hypothesis that in the sand cricket, Gryllus firmus, there are negative genetic correlations between wing morph and reproductive traits. It has previously been shown that in this species the macropterous (flight capable) morph has a delayed age at first reproduction and a decreased total fecundity (Roff, 1984, 1989). Furthermore, the heritability of wing morph is large (approximately 0.65 , Roff, $1986 b$ ), and hence selection for increased or decreased incidence of macroptery should produce rapid changes, a prediction verified by artificial selection (Roff, 1990 b). The existence of genetic correlations between wing morph and reproductive characters was investigated by measuring correlated changes in the age schedule of reproduction during the course of this selection experiment. 
MATERIALS AND METHODS

\section{Experimental protocol}

Details of the species and rearing methods are given in Roff (1986b), and only the salient points are presented here. The stock of $G$. firmus used in the present study was derived from approximately 40 individuals (approximate sex ratio $1: 1$ ) from a single location in northern Florida in 1981. They are maintained in diapause averting conditions $\left(25-30^{\circ} \mathrm{C}\right.$, no set photoperiod but the laboratory lights ensure a relatively long light period), with a breeding stock of between 100-300 individuals. For the selection experiments individuals were raised in batches of 60 individuals per disposable mouse cage, as described in Roff (1986b). Food was provided, ad libitum and comprised Purina ${ }^{(}$ rabbit chow and fresh lettuce leaves.

Previous studies were conducted at $30^{\circ} \mathrm{C}$ and a photoperiod of $17 \mathrm{hrs}$ L: $7 \mathrm{hrs} \mathrm{D}$ : under these conditions the proportion of macropterous males and females is about 76 per cent and 64 per cent, respectively. To reduce this percentage to around $50 \%$ in the females, in the present experiment crickets were reared at $28^{\circ} \mathrm{C}, 15 \mathrm{hrs} \mathrm{L} ; 9 \mathrm{hrs} \mathrm{D}$. The first selected lines were initially constructed as follows: eggs were obtained from the stock culture and the nymphs raised under the experimental conditions. From this group 20 pairs were extracted comprising ten pairs $\mathrm{LW} \times \mathrm{LW}$ (macropterous $\times$ macropterous) and ten pairs $\mathrm{SW} \times \mathrm{SW}$ (micropterous $\times$ micropterous). This procedure was adopted to obtained an initial estimate of the heritability of wing dimorphism (Roff, 1990 b).

To establish a macropterous line (hereafter referred to as the LW line) 200 adults ( 100 males, 100 females) from the ten $\mathrm{LW} \times \mathrm{LW}$ matings were mixed together, with approximately equal representation from each family. Similarly, a micropterous line (SW) was started by mixing together the offspring from the $\mathrm{SW} \times \mathrm{SW}$ crosses. A Control line (C) was formed by mixing the offspring from all crosses. For each of the lines, six cages containing 60 newly hatched nymphs per cage were established. Males and females of a desired morph were selected upon eclosion into adults until approximately 100 of each sex were obtained. The relatively large number of parents was used to prevent inbreeding depression. The mass selection procedure outlined above was followed on all generations subsequent to the first. To provide a replicate, a second series of lines was set up some months after the first, the protocol followed being identical except that a mass selec- tion design was followed from the outset and no initial group of single pair matings constructed.

To estimate the age schedule of fecundities individual pairs were set up as described in Roff (1984). Adults were fed Purina ${ }^{\circledR}$ rabbit chow and eggs removed weekly for a period of four weeks. In pairs from the lines selected for macroptery both males and females were macropterous, while in the pairs from the micropterous lines both adults were micropterous. For the control lines, the male and female within a pairing were of the same morph, one half of the pairings comprising both macropterous adults and one half comprising both micropterous adults. Approximately 20 replicates were used for each combination, the exact number depending upon numbers emerging, space and labour limitations. The fecundity of the micropterous females in the LW lines and the fecundity of the macropterous females in the SW lines were not measured because by generation 5, when fecundity trials were initiated, there were too few to obtain a sufficient sample: as explained below this does not interfere with the statistical analysis. In the first selection experiment, measurements were made at generations 5 and 15 , and in the second experiment at generations 5, 6 and 11 . The total sample size was 419 , comprising 216 macropterous and 203 micropterous females. In addition to weekly egg counts, daily counts to day 14 were made in generation 11 of the second selection experiment.

\section{Statistical analysis}

The measurement of the generic correlation between a threshold trait and some other character presents particular problems. To understand these, first consider the usual situation of two continuously varying characters $\mathrm{X}$ and $\mathrm{Y}$ (e.g., body and size and fecundity) for which a phenotypic relationship exists (fig. 1). Suppose $\mathrm{X}$ is increased or decreased by artificial selection; if the genetic correlation, $r_{\mathrm{g}}$, between $\mathrm{X}$ and $\mathrm{Y}$ is 1 then the correlated trait $Y$ will "slide" along the regression to the point $\mathrm{A}$, but if $r_{\mathrm{g}}$ equals 0 there will be no change in $\mathrm{Y}$ with a change in $\mathrm{X}$ (point $\mathrm{B}$ in fig. 1). If $r_{\mathrm{g}}$ lies between 0 and 1 trait $\mathrm{Y}$ will respond by moving to a point between $\mathrm{A}$ and $\mathrm{B}$.

For a threshold trait we can distinguish only discrete phenotypes, which are, in this case, the macropterous (LW) and micropterous (SW) morphs. However, we assume that there is an underlying variable that shows continuous variation, genotypes exceeding the threshold value producing one phenotype and those below the 


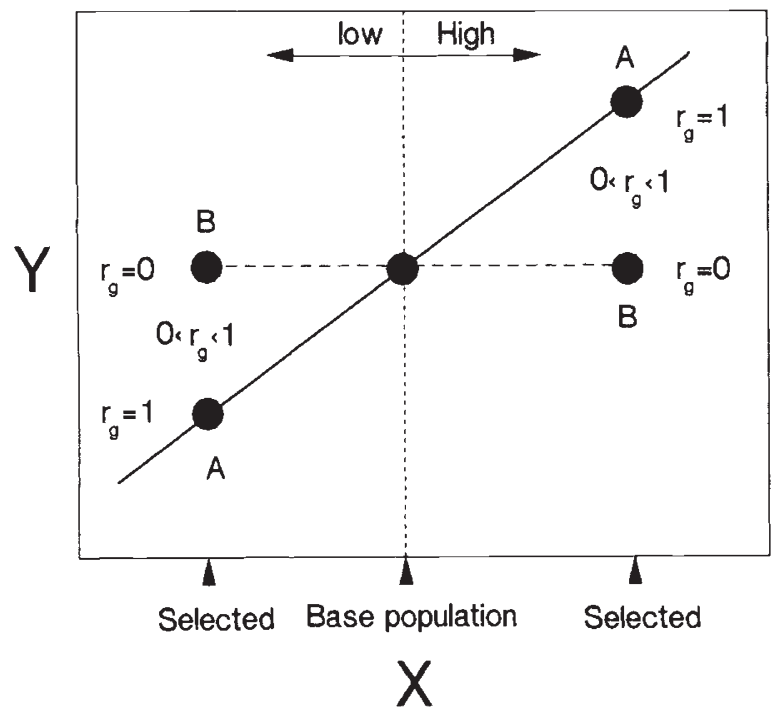

Figure 1 Hypothetical phenotypic relationship between two continuously varying characters $\mathrm{X}$ and $\mathrm{Y}$ (e.g., body size fecundity). If the genetic correlation, $r_{g}$, equals 1 selection on $\mathrm{X}$ will produce a correlated response to $\mathrm{Y}$ to $\mathrm{A}$. If $r_{\mathrm{g}}=0$ there will be no response (B), otherwise $Y$ will move to a point between $A$ and $B$.

threshold producing the alternate (Falconer, 1981). Because it is not possible to measure the actual value of the underlying factor, $\mathrm{X}$, an unambiguous plot of the phenotypic relationship between $X$ and $\mathrm{Y}$ in the unselected population is not possible. If the relationship is linear we can plot three points along the proposed line, two corresponding to the mean values for the two morphs within the unselected population and the third corresponding to the mean value of the whole population (fig. 2(a)). On the other hand, the relationship between $\mathrm{X}$ and $\mathrm{Y}$ might itself correspond to a threshold trait, in which case the mean value for the base population will not, in general, lie on the curve (fig. 2(b)). In the first case, if $r_{\mathrm{g}}=1$, artificial selection for increased per cent LW will decrease $Y$ (e.g., fecundity), while selection for increased per cent $\mathrm{SW}$ will increase $Y$ (points $A$ in fig. 2(a); note that this result is the same as shown in fig. 1). However, in the second scenario there will be no change in $Y$ for a particular morph (fig. 2(b)) though the mean value of $\mathrm{Y}$ for the population as a whole will change as the frequency of each morph changes. We can conclude that if the value of $\mathrm{Y}$ of the $\mathrm{LW}$ morph in the line selected for increased frequency of LW either remains the same or decreases then there is a correlated response and $r_{\mathrm{g}}$ is greater than 0 : a similar argument can be applied to the SW morph in the line selected for increased frequency of SW. The two scenarios can be distinguished by measuring $\mathrm{Y}$ at several generations during selection. Note that even if the value of $\mathrm{Y}$ for the $\mathrm{LW}$ and SW morphs stay the same under selection, the mean value of $\mathrm{Y}$ in the selected population will change by virtue of the changing frequencies of macropterous and micropterous individuals.

The basic statistical model used in the present analysis was,

$$
\begin{aligned}
Y= & a+b X_{1}+c X_{2}+d X_{3} \\
& + \text { interaction terms } \\
& + \text { error }
\end{aligned}
$$

where $X_{1}$ is "wing morph" (LW or SW), $X_{2}$ is "treatment" (selected or control), $\mathrm{X}_{3}$ is "trial", and a, b, c, d are constants. Effects due to "trial" may reflect systematic differences resulting from selection or "error" differences due to "random" variation between trials; for this reason I used a categorical variable for "trial".

\section{RESULTS}

The response to selection was very rapid, and by generation 5 the frequency of macropterous crickets had risen to over 80 per cent in the $\mathrm{LW}$ lines and decreased to less than 10 per cent in the SW lines (fig. 3). An asymmetrical response was obtained with the incidence of microptery increasing more rapidly in the $\mathrm{SW}$ lines than the incidence of macroptery in the LW lines (fig. 3, Roff, 1990b). Given the large changes in frequency, if a correlated response in fecundity and/or age at first reproduction exists, it should be evident by generation 5 when the first measurements were made.

The overall mean egg production in the first week was $77 \cdot 4(\mathrm{SE}=6.6)$ eggs for the micropterous females and $39.5(5.5)$ for the macropterous females. Many females did not lay eggs in the first week and hence for this week a logarithmic transformation (log $\{$ eggs +1$\}$ ) was used (the analysis was also done with the untransformed data without significant differences in the results). In week 1 there was a significant effect of wing morph $\left(X_{1}\right)$ and trial $\left(\mathrm{X}_{3}\right)$, but no effect of treatment $\left(\mathrm{X}_{2}\right.$, table $1)$, and no significant interaction effects. The significant effect due to trial was due to the fecundities of females in all lines at generation 15 of the first selection experiment being low, a reduction that was also found in the remaining three weeks. While inbreeding depression cannot be entirely ruled out, the large number of males and females contributing to each generation, and the fact that there has been 

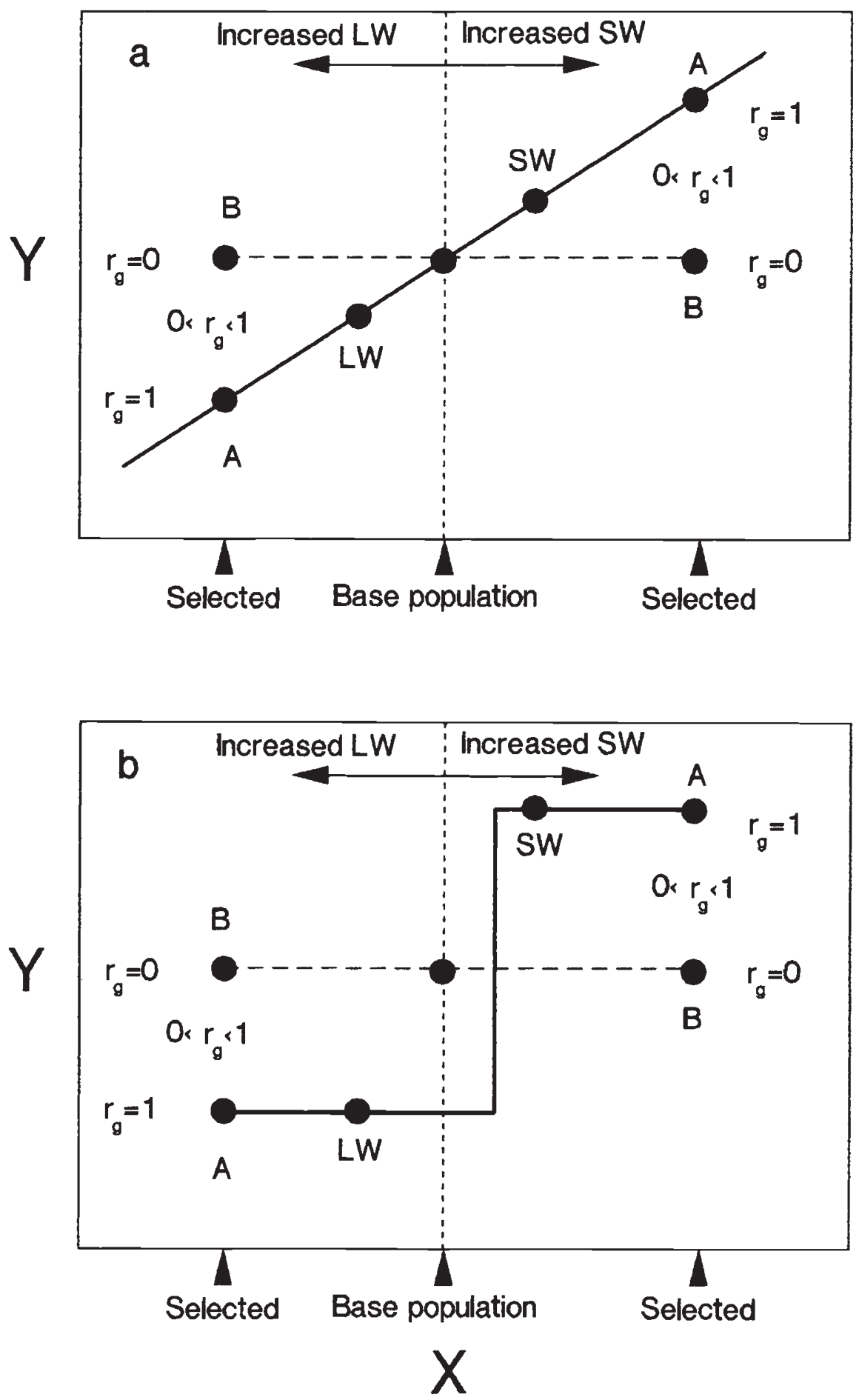

Figure 2 Two hypothetical phenotypic relationships between a continuously varying character, $X$, which determines the expression of a threshold character (e.g., wing dimorphism) and a second character $Y$ (e.g., fecundity). The average value of the two morphs (LW and SW) will lie on the line but the mean value for an individual from the population need not do so (e.g., panel b). If $r_{\mathrm{g}}=1$ selection for a change in the incidence of macroptery will cause a correlated response of $\mathrm{Y}$ to $\mathrm{A}$ : if $r_{\mathrm{g}}=0$, the mean value of the population will remain constant (B), otherwise $L W$ and $S W$ will move to values between $A$ and $B$. 
no obvious reduction in hatchling numbers during the selection experiments, suggests that this reduction was due to some other cause.

The above results indicate, (1) that the fecundity of a macropterous or micropterous female did not depend upon whether it came from a selected line or the control line, and (2) that the SW morph lay significantly more eggs than the LW morph. Thus, the mean 1st week fecundity of the LW line decreased as the incidence of macroptery increased, while that of the SW line increased as the incidence of microptery increased. The lack of a significant interaction term suggests that the relationship between fecundity and the factor controlling wing morph is more like a threshold trait (fig. 2(b)) than a continuous linear function (fig. 2(a)).

The increased fecundity of the SW morph could be due to a decrease in the age at first reproduction and/or a higher rate of egg production. The first possibility was tested in two ways. First, I compared the proportion of females (arcsine transformed) that began oviposition in the first week (i.e., fecundity $>0$ ) with respect to wing morph, treatment and trial. As in the previous analysis there was a significant effect of wing morph $(P=$ 0.04 ; i.e., more micropterous females layed eggs in the first week than macropterous females), and trial $(P=0 \cdot 001)$, but no effect of treatment $(P=$ 0.52 ), and no significant interaction effects. Secondly, I compared the ages at first reproduction obtained from the daily egg counts made on line 2 at generation 11 (fig. 4). The statistical model used was, $Y=a+b X_{1}+c X_{2}+d_{1} X_{2}+$ error, where $Y=$ day at first reproduction, $X_{1}=$ wing morph, $\mathrm{X}_{2}=$ treatment, and $\mathrm{a}, \mathrm{b}, \mathrm{c}, \mathrm{d}$ are fitted constants. There was no significant interaction $\left(F_{1,94}=4 \cdot 59\right.$,
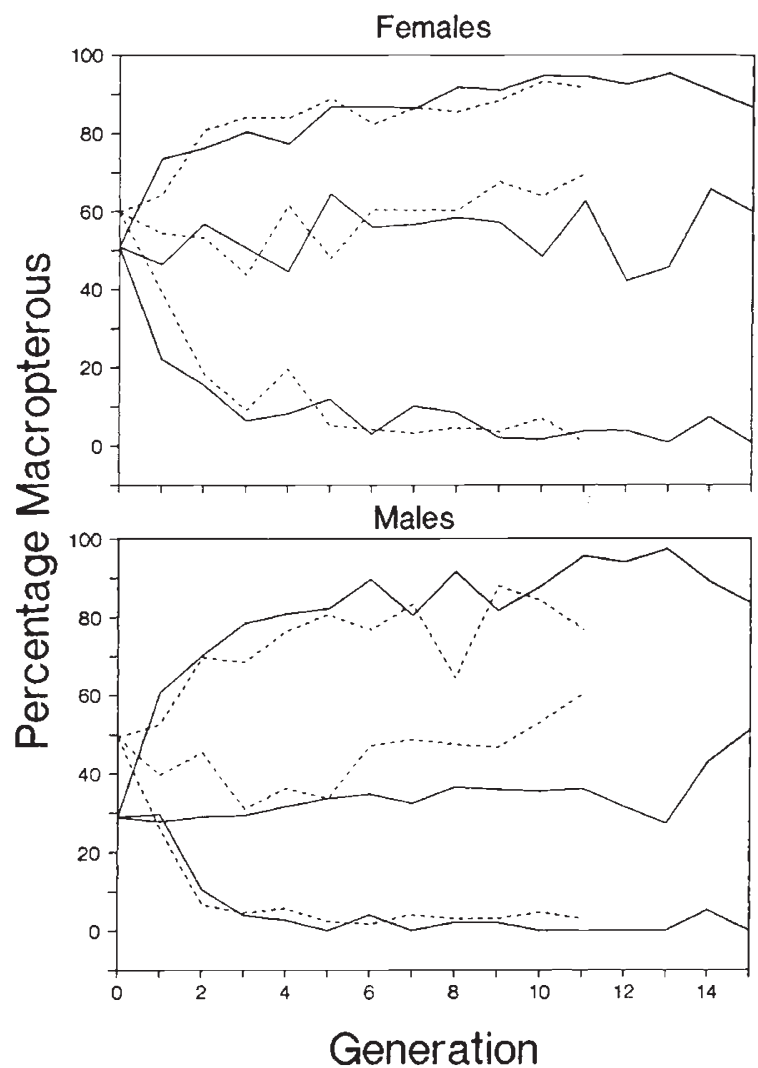

Figure 3 Response to selection for increasing and decreasing incidence of macroptery in Gryllus firmus. Line 1 -; Line 2 - - -

$P>0.05)$ and no significant effect of treatment after adjusting for wing morph $\left(F_{1,94}=0 \cdot 14, \mathrm{P}>\right.$ $0 \cdot 5)$, but a highly significant effect of wing morph $\left(F_{1,94}=16 \cdot 5, P<0.0001\right)$. The mean age at first

Table 1 Correlation coefficients for the regressions of fecundity on wing morph, treatment and trial (cols. 1-3). Because there was no effect of treatment only the adjusted $R$ value of the additive model comprising $X_{1}$ and $X_{3}$ (col. 4), and the saturated model $\left(X_{1}, X_{2}\right.$, $X_{3}, X_{1} X_{2}, X_{1} X_{3}, X_{2} X_{3}, X_{1} X_{2} X_{3}$ ) are presented. Total sample size is 419 .

\begin{tabular}{|c|c|c|c|c|c|}
\hline \multirow[b]{2}{*}{ Week } & \multicolumn{4}{|l|}{ Model } & \multirow[b]{2}{*}{ Saturated } \\
\hline & $X_{1}$ & $\mathrm{X}_{2}$ & $\mathrm{X}_{3}$ & $X_{1}, X_{3}$ & \\
\hline Week $1^{\text {a }}$ & $-0.23^{* *}$ & 0.00 & $0 \cdot 30^{* *}$ & $0.37 * *$ & $0 \cdot 37^{* *}$ \\
\hline Week 2 & 0.00 & 0.00 & $0.33^{* *}$ & $0.33^{* *}$ & $0.37^{* *}$ \\
\hline Week 3 & $0.11^{*}$ & 0.00 & $0 \cdot 36^{* *}$ & $0.38 * *$ & $0.37^{* *}$ \\
\hline Week 4 & $0.11^{*}$ & 0.00 & $0.20^{* *}$ & $0.24^{* *}$ & $0.23^{*}$ \\
\hline Total & 0.00 & 0.00 & $0 \cdot 36^{* *}$ & $0.36^{* *}$ & $0.35^{* *}$ \\
\hline
\end{tabular}

$\mathrm{X}_{1}$ : Wing morph (SW or LW).

$\mathrm{X}_{2}$ : Treatment (Selected or Control).

$\mathrm{X}_{3}$ : Trial (6 trials).

a: based on $\log (x+1)$ transformation.

${ }^{*} P<0.01$. ${ }^{* *} P<0.001$. 


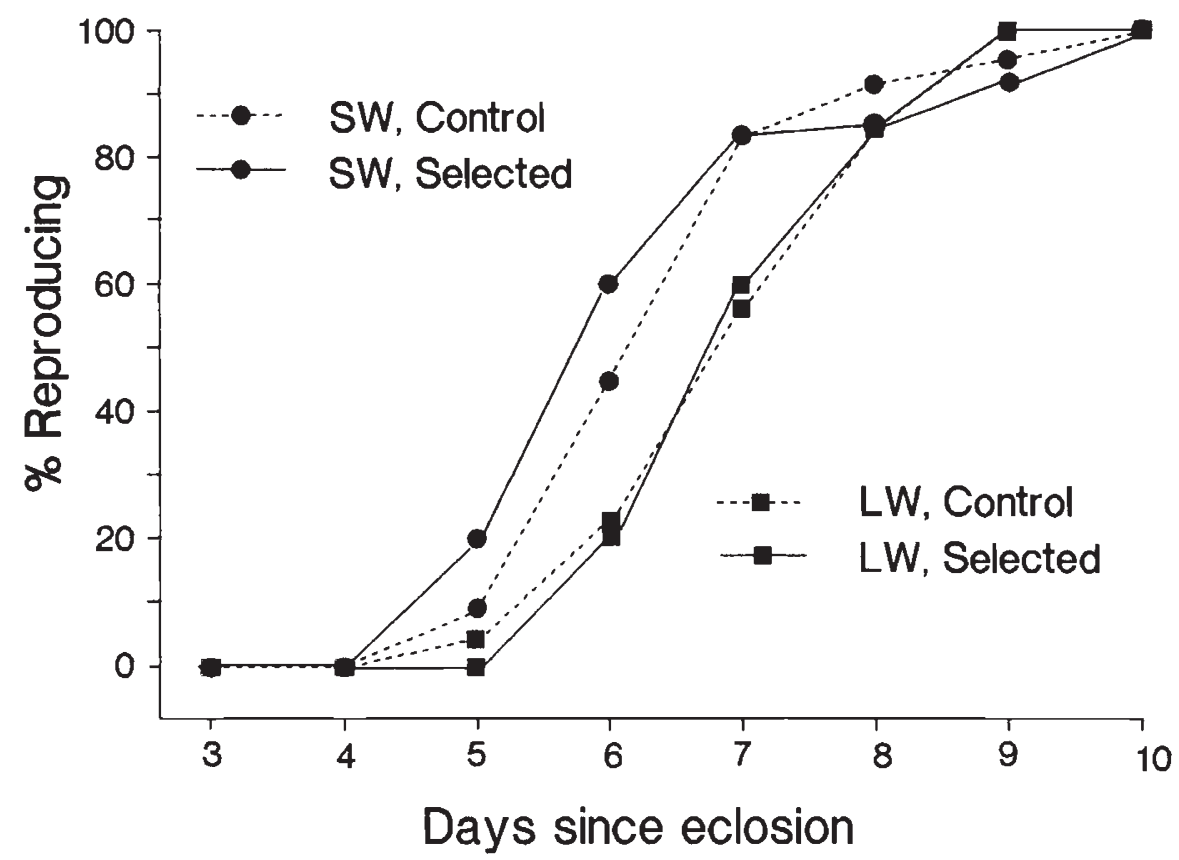

Figure 4 Cumulative proportion of females laying as a function of age since eclosion in G. firmus.

reproduction of micropterous females was 6.55 days $( \pm 0 \cdot 14)$ and for macropterous females $7 \cdot 45$ days $( \pm 0 \cdot 18)$. These two analyses indicate, (1) that micropterous female crickets begin reproducing at an earlier age than macropterous crickets, and (2) that this difference is maintained under selection for changes in the incidence of macroptery. They do not rule out the possibility of additional differences in rate of egg production.

Egg production in the second week was not correlated with wing morph or treatment, but was significantly correlated to trial (table 1). Ignoring the effects of trial, the overall mean number of eggs laid was $389 \cdot 8(26 \cdot 5)$ for micropterous females and $366.0(18.15)$ for macropterous. In weeks 3 and 4 there was a significant effect of wing morph on egg production (table 1) but, contrary to egg production in week 1 , macropterous females produced more eggs than micropterous females (in week 3 micropterous females laid $345 \cdot 0$ [19.5] eggs compared to 412.4 [ 19.74$]$ laid by macropterous females: results for week 4 were 286.9 [16.5] and $348 \cdot 1$ [17.33], respectively). Furthermore, in contrast to the results obtained at $30^{\circ} \mathrm{C}$ and a photoperiod of $17 \mathrm{~L}: 7 \mathrm{D}$, the total egg production of micropterous females was not significantly different from that of macropterous females (table 1 , means of 1099 [54.0] and 1166 [47.28] for micropterous and macropterous females, respectively).
In summary, differences in the age schedule of reproduction were found between wing morphs but not between crickets of the same morph from different lines. The micropterous morph had a higher egg production in the first week after eclosion than the macropterous morph but the total fecundity of the two morphs was the same. The higher egg production in the first week of the micropterous morph was, in part at least, a consequence of an earlier age at first reproduction.

The maintenance of differences in the age schedules of reproduction between micropterous and macropterous crickets means that as the frequency of the macropterous morph changes in response to selection on the incidence of macroptery there will be a correlated response in the mean age schedule of reproduction in the population. Thus we can conclude that the phenotypic relationship between the age schedule of reproduction and wing morph observed in the unselected population is in part due to a genetic correlation between the traits.

\section{DISCUSSION}

The micropterous morph of $G$. firmus begins reproducing after eclosion sooner than the macropterous morph and lays more eggs in the first week of adult life. The results of the selection experiment 
indicate that these differences in reproduction are genetically correlated to wing morph. Contrary to the finding at $30^{\circ} \mathrm{C}$ (Roff, 1984, 1989), when the crickets are raised at $28^{\circ} \mathrm{C}$ there is no difference in total fecundity between the two morphs. The absence of a difference is caused by a "crossingover" of the $m_{\mathrm{x}}$ curves (i.e., the age schedules of reproduction) in the second week. Such an intersection was noted by Fairbairn (1988) for Gerris remigis, Limnoporus caniculatus, (data from Zera, 1984), and the cricket species, Gryllus firmus and Allonemobius fasciatus, (data from Roff, 1984). It also occurs in Javesella pellucida (Mochida, 1973), Cavelerius saccharivorus (Fujisaki, 1986), and Horvathiolius gibbicollis (Solbreck, 1986), but not in the aphid species Setobion avenae, Metopolophium dirhodum (Wratten, 1977) and Aphis fabae (Dixon and Wratten, 1971). A reversal in egg production later in adult life does not necessarily mean that the egg production of both morphs will eventually be equal; for example, the intersection of the $m_{x}$ curves in $G$. firmus at $30^{\circ} \mathrm{C}$ occurs late in reproductive life and could not lead to the cumulative fecundity of the LW morph eventually catching up to that of the SW (Roff, 1984, 1989). An intersection of the $m_{\mathrm{x}}$ curves may be typical in wing dimorphic species, but this does not generally offset the fecundity advantage of the SW morph (Roff, 1986a; Roff and Fairbairn, 1990). That the fecundity advantage is negated under one environmental regime but not another in $G$. firmus suggests that more data are needed on the relationship between the age schedule of reproduction and different environmental conditions among insect species.

The fact that the difference in fecundity between the same morphs from the selected and control lines did not change in any systematic way with the generation of selection suggests that the relationship between $m_{\mathrm{x}}$ and the factor controlling the expression of wing form is more like a threshold function (fig. 2(b)) than a linear function (fig. 2(a); the lack of a change in the selected lines with generation cannot be used as sole evidence since after generation 5 little further response to selection occurred: the important observation is the lack of a difference between selected and control lines). Because there was significant variation between trials which may have obscured small changes this conclusion must be considered tentative. It is not unreasonable to postulate a threshold type of response in the present case if the wing form delineates a migratory and non migratory morph. However, interspecific comparisons of the flight propensity of various gerrid species and an assay of flight propensity in the selected and control stocks of $G$. firmus suggest that even among the macropterous morph there exists a continuum of migratory propensity (Fairbairn, 1986; Butler, 1987; Fairbairn and Desranleau, 1987; Fairbairn and Roff, 1990). Therefore, it would be premature to presume a threshold response with respect to $m_{\mathrm{x}}$ : further experiments using lines that are vitually pure breeding and larger sample sizes are required to examine this question.

In the laboratory culture there is clearly no advantage to retaining flight capability for migration; therefore, why is the morph maintained under laboratory culture? The proportion of micropterous males and females in the stock culture are presently 97.3 per cent and 80.56 per cent, respectively. Since the percentage of each morph in the stock culture has not been monitored it is possible that the incidence of macroptery has declined. However, since neither the temperature nor photoperiod are monitored the high incidence of microptery may be due to environmental conditions. Recent extractions from the stock indicate that the incidence of macroptery under the temperature and photoperiod conditions of the selection experiment produce aproximately 50 per cent macroptery, as found at the initiation of the selection experiment (Roff, unpublished data). A second reason why macroptery may be maintained is that the fecundity advantage of micropterous crickets may disappear at lower temperatures. The present experiments indicate that the cumulative fecundity advantage of micropterous females is found at $30^{\circ} \mathrm{C}$ but not at $28^{\circ} \mathrm{C}$, though this could be a consequence of differences in temperature and/or humidity since the experiments were run in different incubators. Data presently being analysed also suggest that macropterous crickets develop faster than micropterous crickets at lower but not higher temperatures: the shorter development time of the macropterous morph could counterbalance the longer period between final eclosion and first reproduction. Furthermore, there may be no fitness advantage to microptery in the male sex (Roff and Fairbairn, 1990). These factors, the environmental conditions of culture, a change in relative fitness with environmental factors and a lack of difference in fitness between the morphs within the males, will at least slow down the rate at which the micropterous morph will be expected to increase in frequency. The consequences of these factors on the relative fitness of the two morphs is under study.

The hypothesis that there are trade-offs between traits is central to most theories of the 
evolution of life history variation (Stearns, 1976, 1977; Maynard Smith, 1978). The experiment presented in this paper demonstrates that, in principle, life history variation in wing dimorphic insects may be generated by the genetically determined tradeoff between migratory ability and reproduction. Macropterous individuals can migrate and hence the loss of fitness accruing from a delayed age at first reproduction and, under some conditions a reduced fecundity, is compensated by the advantage of migration in an ephemeral habitat. The genetic correlation between these two traits means that, at least in the short term, evolutionary changes in one will be modulated by the decreased fitness necessarily induced by the other (antagonistic pleiotropy).

In some species, such as Pyrrhocoris apterus (Honěk, 1976), Gerris remigis (Fairbairn, 1986) and Chorthippus parallelus (Ritchie et al., 1987), the macropterous morph is found only at very low frequencies and does not fly. Ritchie et al. (1986) speculated that the macropterous form of Chorthippus parallelus, though showing the same delay in reproduction and cumulative fecundity as other wing dimorphic species, may possess fitness advantages other than the ability to disperse. However, Fairbairn (1986) argued that in G. remigis populations from Eastern Canada the occurrence of macropters is a result of unusual environmental conditions and not a balance between fitness components of the apterous and macropterous morphs: this mechanism could also account for the occassional appearance of macropterous Chorthippus parallelus (Ritchie, personal communication). Selection against the appearance of non migratory macropterous forms at low frequency would be very weak and macroptery could persist for a long time as a "genetic relic": it should not be supposed that all populations are at genetic equilibrium (Berven and Gill, 1983; Fairbairn, 1986; Fairbairn and Roff, 1990). Macroptery is not a "genetic relic" in G. firmus, the incidence of macroptery in the field being as high as 23 per cent (Veazey et al., 1976) and the macropterous form being capable of, and willing to fly (Fairbairn and Roff, 1990; Roff, personal observation). For this species, for other North American Gryllus species and North American Orthopera in general, the hypothesis that the dimorphism is maintained, in part at least, by environmental heterogeneity appears to be the most attractive at present (Walker and Sivinski, 1986; Roff, 1990c).

Antagonistic pleiotropy is important not only because it can determine the optimal combination of traits but because it may also play a role in preserving genetic variation (reviewed in Rose, 1983, 1985). In single locus models some dominance is required for a genetic polymorphism to be sustained by antagonistic pleiotropy (Rose, 1982). Single locus mechanisms for the determination of wing morph are found in the insects, particularly among those with holometabolous metamorpbosis (Roff and Fairbairn, 1990). It is difficult to evaluate explicitly the relative fitness values of macropterous and micropterous morphs, but simulations using single locus models with dominance demonstrate that in a heterogeneous environment polymorphisms for migration can be preserved as a consequence of the differences in fitness of the two morphs (Roff, 1975). Dominance is also necessary but less important in polygenic systems (Rose, 1985), but without further numerical analysis, it is not possible to define the exact conditions required to maintain variation of the incidence of macroptery in a heterogeneous environment.

Several factors may operate in conjunction with antagonistic pleiotropy to maintain variation. First, the expression of wing form both in G. firmus and wing dimorphic insects in general, is highly dependent on environmental conditions such as temperature and photoperiod (Honěk, 1976; Roff, unpublished data). Therefore, though the heritability of wing form is high under constant conditions (approximately 0.65; Roff, 1986 b, 1990), it may be very low under the highly variable conditions that may occur in the field, and phenotypic differences in wing form and the age schedule of reproduction may not reflect genetic variation. Thus much of the genetic variation may be effectively "hidden" from the action of natural selection. Secondly, the optimal level of migration and hence wing dimorphism may vary between regions; as there will undoubtedly be migration between regions there may exist no level of migration for both regions that is most fit and selection may thus tend to preserve variation, or at least erode it at a very slow rate. Future research must address the question of genotype by environment interactions and the pattern of environmental heterogeneity found in the field.

Acknowledgements I am most grateful for the constructive criticisms of Drs D. J. Fairbairn, H. Dingle, J. Endler, T. Prout and M. Turelli. Catherine Helick and Sharon David provided indispensable technical assistance. This work was supported by an operating grant from National Science and Engineering Council of Canada. 


\section{REFERENCES}

BUTLER, T. 1987. Population dynamics and dispersal in waterstriders: a comparative approach. M.Sc. Thesis, Concordia University, Quebec, Canada.

DENNO, R. F., OLMSTEAD, K. L. AND McClOUd, E. S. 1989. Reproductive cost of flight capability: a comparison of life history traits in wing dimorphic planthoppers. Ecol. Ent., 14, 31-44.

DINGLE, H. 1985. Migration. In Kerkut, G. A. and Gilbert, L. I. (eds), Comprehensive Insect Physiology, Biochemistry and Pharmacology. Vol. 9, Behaviour. Pergamon Press, New York.

DIXON, A. F. G. AND WRATTEN, S. D. 1971. Laboratory studies on aggregation, size and fecundity in the black bean aphid, Aphis fabae Scop. Bull. ent. Res., 61, 97-111.

FAIRBAIRN, D. J. 1986. Does alary dimorphism imply dispersal dimorphism in the waterstrider, Gerris remigis? Ecol. Ent., $11,355-368$.

FAIRBAIRN, D. J. 1988. Adaptive significance of wing dimorphism in the absence of dispersal: a comparative study of wing morphs in the waterstrider, Gerris remigis. Ecol. Ent., 13, 273-281.

FAIRBAIRN, D. J. AND DESRANLEAU, L. 1987. Flight threshold, wing muscle histolysis, and alary polymorphism: correlated traits for dispersal tendency in the Gerridae. Ecol. Ent., 12, 13-24.

FAIRBAIRN, D. J. AND ROFF, D. A. 1990. Genetic correlations among traits determining migratory tendency in the sand cricket, Gryllus firmus. Evolution, (In press).

FALCONER, D. S. 1981. Introduction to Quantiative Genetics. 2nd ed. Longman, London and New York.

FUJISAKI, K. 1986. Reproductive properties of the oriental chinch bug, Cavelerius saccharivorus Okajima (Heteroptera: Lygaeidae), in relation to its wing polymorphism. Res. Pop. Ecol., 28, 43-52.

HARRISON, R. G. 1980. Dispersal polymorphism in insects. $\boldsymbol{A}$. Rev. Ecol. Syst., 11, 95-118.

HONĚK, A. 1976. Factors influencing the wing polymorphism in Pyrrhocoris apterus (Heteroptera, Pyrrhocoridae). Zool. Jb. Syst. Bd., 103, 1-22.

INGLESFIELD, C. AND BEGON, M. 1983. The ontogeny and cost of migration in Drosophila subobscura Collin. Biol. J. Linn. Soc., Lond., 167, 76-96.

JOHNSON, C. G. 1969. Migration and Dispersal of Insects by Flight. Methuen, London.

MAYNARD SMITH, J. 1978. Optimization theory in evolution. A. Rev. Ecol. Syst., 9, 31-56.

MOCHIDA, O. 1973. The characters of the two wing-forms of Javesella pelucida (F.) (Homoptera: Delphacidae) with special reference to reproduction. Trans. $R$. ent. Soc. Lond., $125,177-225$.

RITCHIE, M. G., BUTLIN, R. K. AND HEWITT, G. M. 1987. Causation, fitness effects and morphology of macropterims in Chorthippus parallelus (Orthoptera: Acrididae). Ecol. Ent., 12, 209-218.

ROFF, D. A. 1975. Population stability and the evolution of dispersal in a heterogeneous environment. Oecologia, 19, 217-237.
ROFF, D. A. 1977. Dispersal in dipterans: its costs and consequences. J. Anim. Ecol., 46, 443-456.

ROFF, D. A. 1984. The cost of being able to fly: a study of wing polymorphism in two species of crickets. Oecologia, 63, 30-37.

ROFF, D. A. $1986 a$. The evolution of wing dimorphism in insects. Evolution, 40, 1009-1020.

ROFF, D. A. $1986 \mathrm{~b}$. The genetic basis of wing dimorphism in the sand cricket, Gryllus firmus and its relevance to the evolution of wing dimorphisms in insects. Heredity, 57, 221-231.

ROFF, D. A. 1989. Exaptation and the evolution of dealation in insects. J. evol. Biol., 2, 109-123.

ROFF, D. A. $1990 a$. Understanding the evolution of insect life cycles: the role of genetical analysis. In, Gilbert, F. (ed.), Genetics, Evolution and Coordination of Insect Life Cycles. Springer-Verlag, Berlin (In press).

ROFF, D. A. $1990 \mathrm{~b}$. Selection for changes in the incidence of wing dimorphism in Gryllus firmus. Heredity, 65, 163-168.

ROFF, D. A. $1990 \mathrm{c}$. The evolution of flightlessness in insects. Ecol. Monogr., (In press).

ROFF, D. A. AND FAIRBAIRN, D. J. 1990. Wing dimorphisms and the evolution of migratory polymorphisms among the Insecta. Am. Zool., (In press).

ROSE, M. R. 1982. Antagonistic pleiotropy, dominance and genetic variation. Heredity, 48, 63-78.

ROSE, M. R. 1983. Theories of life-hostory evolution. Am. Zool., $23,15-23$.

ROSE, M. R. 1985. Life history evolution with antagonistic pleiotropy and overlapping generations. Theor. Pop. Biol., $28,342-358$.

SOLBRECK, C. 1986 . Wing and flight muscle polymorphism in a lygaeid bug, Horvathiolus gibbicollis: determinants and life history consequences. Ecol. Ent., 11, 435-444.

SOUTHWOOD, T. R. E. 1962. Migration of terrestrial arthropods in relation to habitat. Biol. Rev., 37, 171-214.

STEARNS, S. C. 1976. Life-history tactics: a review of the ideas. Q. Rev. Biol., 51, 3-47.

STEARNS, S. C. 1977 . The evolution of life-history traits: a critique of the theory and a review of the data. Ann. Rev. Ecol. Syst., 8, 145-171.

VEAZEY, J. N., KAY, C. A. R., WALKER, T. J. AND WHITCOMB, W. H. 1976. Seasonal abundance, sex ratio, and macroptery of field crickets in Northern Florida. Ann. ent. Soc. Amer., 69, 374-380.

WALKER, T, AND SIVINSKI, J. M. 1986. Wing dimorphism in field crickets (Orthopera: Gryllidae: Gryllus). Ann. ent. Soc. Amer., 79, 84-90.

WRATTEN, S. D. 1977. Reproductive strategy of winged and wingless morphs of the aphids Setobion avenae and Metopolophium dirhodum. Ann. appl. Biol., 85, 319-331.

ZERA, A. J. 1984. Differences in survivorship, development rate and fertility between the long-winged and wingless morphs of the waterstrider, Limnoporus canaliculatus. Evolution, 38, 1023-1032. 